\title{
Eccentricity Boost of Stars Around Shrinking Massive Black Hole Binaries
}

\author{
Mao Iwasa and Naoki Seto \\ Department of Physics, Kyoto University Kyoto 606-8502, Japan
}

(Dated: August 10, 2021)

\begin{abstract}
Based on a simple geometrical approach, we analyze the evolution of the Kozai-Lidov mechanism for stars around shrinking massive black hole binaries on circular orbits. We find that, due to a peculiar bifurcation pattern induced by the Newtonian potential of stellar clusters, the orbit of stars could become highly eccentric. This transition occurs abruptly for stars with small initial eccentricities. Our approach would be also useful for studying the Kozai-Lidov mechanism in various astrophysical contexts.
\end{abstract}

\section{INTRODUCTION}

Coalescence of a massive black hole $(\mathrm{MBH})$ binary is a spectacular event and a huge amount of energy is converted into gravitational waves (GWs). Depending on the masses of the binary, the planned space interferometers such as eLISA [1] can detect the strong GW signal, even from a very high redshift. Meanwhile, associated with shrinkage of $\mathrm{MBH}$ binaries in galactic nuclei, the infall rate of stars (more precisely, stellar-mass objects) to the MBHs could be enhanced significantly [2]. These stars would emit GWs or electromagnetic waves that encode precious information for astrophysics, cosmology and fundamental physics (e.g. strong gravity) 3].

For the infall into the MBHs, the star must have an eccentricity close to unity, and it has been actively discussed that the Kozai-Lidov (KL) mechanism could play important roles [4 [6]. In the KL mechanism, the inner eccentricity of a hierarchical triple system increases due to the exchange of inner and outer angular momenta [7].

In this paper, concentrating on the MBH inspirals, we theoretically examine the time evolution of the hierarchical triple systems that specifically satisfy the following two conditions; (1) the KL mechanism is initially suppressed by the inner apsidal precessions (due to nonKeplerian potentials [8-10]), but (2) it gradually becomes effective, along with the slow (to be more precise, adiabatic) contraction of the outer orbits (see also [11, 12]).

Our primary aim is to develop a simple geometrical approach for the adiabatic evolution of the KL mechanism (see also [13] for similar studies mean-motion resonances). More specifically, we analyze the phase-space structure of the corresponding time-dependent Hamiltonian, and concurrently apply the arguments of the adiabatic invariance. This approach would be complementary to numerical studies, for understanding the KL mechanism of the triple systems including $\mathrm{MBH}$ binaries. The KL mechanism has been also applied to various astrophysical phenomena [8 10, 14 20], and our approach could be useful for studying them.

This paper is organized as follows. In section II, we analyze the hierarchical systems composed purely by three point-masses, nevertheless including relativistic effects.
These simple systems are suitable not only for explaining our geometrical approach but also for numerically examining its validity. Then, in section III, we apply our approach to individual stars in a stellar cluster associated with slowly shrinking $\mathrm{MBH}$ binaries. We find that the eccentricities of the stars show intriguing evolutions. These results could be well explained by a peculiar bifurcation pattern caused by the Newtonian potential of the stellar cluster. We also discuss the effects of relaxation processes for our geometrical approach.

\section{KL MECHANISM FOR PN SYSTEMS}

\section{A. Numerical Experiments}

We first examine the evolution of relativistic hierarchical triple systems, only including gravitational interaction with the Post-Newtonian (PN) approximation. This is for demonstrating our geometrical approach and also providing an example in distinction from the Newtonian effects of a stellar cluster (discussed in section III).

Our triple systems are made from two spinless MBHs (masses: $\left.m_{0}, m_{2}\right)$ and a stellar mass object $\left(m_{1}\right)$. The inner binary is composed by $m_{0}$ and $m_{1}$, while the remaining $\mathrm{MBH} m_{2}$ is the outer tertiary. Below, we use the geometrical unit with $c=G=m_{0}+m_{1}+m_{2}=1$ and the labels $j \in\{1,2\}$ for the inner and outer orbital elements. We denote the semimajor axes by $a_{j}$, the eccentricities by $e_{j}$, the arguments of pericenters by $g_{j}$ and the inclination between the inner and outer orbits by $I$. For the inner angular momentum, we also define

$$
G_{1} \equiv \sqrt{1-e_{1}^{2}}
$$

and

$$
J_{1} \equiv G_{1} \cos I
$$

(the component normal to the outer orbital plane, $J_{1} \leq$ $\left.G_{1} \leq 1\right)$. To simplify our arguments, we fix $m_{0}=0 . \overline{3}$, $m_{1}=0.3 \times 10^{-6}$ and $m_{2}=0.7$

To begin with, we make numerical simulations for the relativistic triple systems. For the direct three-body calculations, we use the equations of motion derived from 
the 2.5PN ADM Hamiltonian [21], formally expressed as

$$
H_{\mathrm{ADM}}=H_{\mathrm{N}}+H_{1 \mathrm{PN}}+H_{2 \mathrm{PN}}+Y H_{2.5 \mathrm{PN}}
$$

Here $H_{\mathrm{N}}, H_{1 \mathrm{PN}}$ and $H_{2 \mathrm{PN}}$ are the Newtonian, $1 \mathrm{PN}$, and $2 \mathrm{PN}$ terms. The last one $H_{2.5 \mathrm{PN}}$ is the leading dissipative term and $Y$ is an artificial parameter to speed up our numerical calculations.

Using a fourth-order Runge-Kutta scheme with $Y=$ $10^{4}$ 1, we numerically solve the evolution of the following three systems, s-I, s-II and s-III. Their initial conditions are $\left(e_{1, \mathrm{i}}, a_{2, \mathrm{i}}, e_{2, \mathrm{i}}\right)=(0.001,6300,0.0001)$ for $\mathrm{s}-$ I, $(0.001,6300,0.3)$ for s-II and $(0.3,5700,0.0001)$ for s-III. Other orbital elements are commonly set at $a_{1, \mathrm{i}}=400$, $g_{1, \mathrm{i}}=\pi / 2$ and $g_{2, \mathrm{i}}=0$. We also choose the initial inclination $I_{\mathrm{i}}$ to realize $J_{1, \mathrm{i}} \equiv \sqrt{1-e_{1, \mathrm{i}}^{2}} \cos I_{\mathrm{i}}=0.150\left(I_{\mathrm{i}} \sim 80^{\circ}\right.$ for our systems). Our main targets are s-I and s-III with different initial inner eccentricities $e_{1, i}$. The system $\mathrm{s}-\mathrm{II}$ is for briefly studying effects of the outer eccentricities $e_{2, i}$. Note also that we set the above orbital parameters for demonstration of our geometrical approach. More realistic systems (but more complicated for direct calculations) would be analyzed in the next section.

We terminate our calculations, when the instantaneous separation between $m_{0}-m_{1}$ becomes smaller than $10 m_{0}$. All the three systems are dynamically stable until this termination condition (satisfying the stability criterion of [22]).

Here, we summarize the characteristic timescales. For the outer orbits of s-I and s-III, we have the merger time $T_{2, \mathrm{GW}} \simeq 4.82 \times 10^{10}\left(a_{2} / 6000\right)^{4}\left(10^{4} / Y\right)$ due to $\mathrm{GW}$ emission [23]. In the following, we use the decreasing distance $a_{2}$ as an effective time variable. Meanwhile, in the range $a_{2} \geq 4000$ relevant for the analysis below, we can neglect the radiation reaction for the inner orbits with $T_{1, \mathrm{GW}}>60 T_{2, \mathrm{GW}}$. The timescales of the KL mechanism and the PN apsidal precession are also important for the inner binary. Their typical values are

$$
\begin{aligned}
T_{\mathrm{KL}} & \equiv \frac{2}{3 \pi} \frac{\left(m_{0}+m_{1}+m_{2}\right)}{m_{2}} \frac{P_{2}^{2}}{P_{1}}, \\
T_{1 \mathrm{PN}} & \equiv \frac{a_{1}^{5 / 2}\left(1-e_{1}^{2}\right)}{3\left(m_{0}+m_{1}\right)^{3 / 2}},
\end{aligned}
$$

where $P_{1}$ and $P_{2}$ are the inner and outer orbital periods [8]. As we have $T_{2, \mathrm{GW}} \gg T_{\mathrm{KL}}, T_{1 \mathrm{PN}}$ for our three systems, the outer orbits shrink adiabatically. Also, the KL mechanism is initially suppressed by the inner relativistic precession with $T_{\mathrm{KL}}>T_{1 \mathrm{PN}}$.

Next, we describe our numerical results for s-I and s-III. As expected, the shrinkages of $a_{1}$ are small with $\left|\delta a_{1} / a_{1, \mathrm{i}}\right|<0.04$ down to the epoch of $a_{2}=4000$ (fluctuations of $\left.J_{1}:\left|\delta J_{1} / J_{1, \mathrm{i}}\right|<0.1\right)$. Figure. 1 represents the

1 We obtained almost the same results with $Y=500$, by dropping the time-consuming $2 \mathrm{PN}$ term.

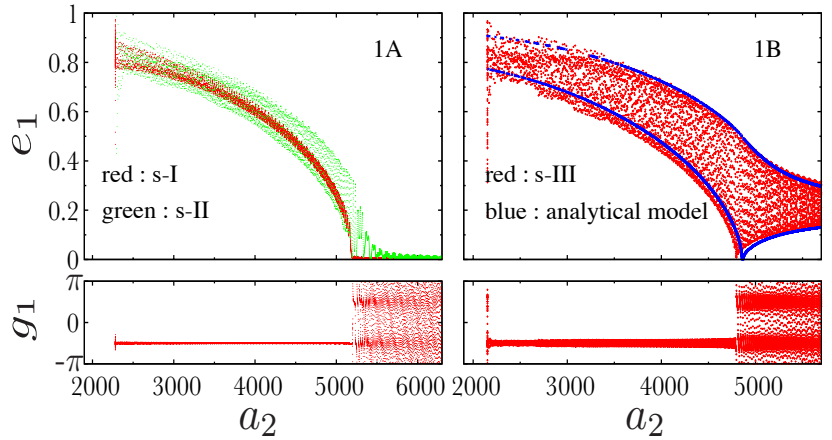

FIG. 1. Evolution of the inner orbital elements $e_{1}$ and $g_{1}$, as a function of decaying $a_{2}$. Panel 1A: results from the direct three-body calculations for s-I (red points) and s-II (green points). Panel 1B: results from the direct three-body calculation for s-III (red points) and the corresponding analytical predictions (blue points) for the minimum and maximum of $e_{1}$ at each $a_{2}$.

evolution of $e_{1}$ and $g_{1}$ as a function of $a_{2}$. In the case of s-I, $e_{1}$ suddenly starts to increase at $a_{2} \sim 5200$ (showing the characteristic feature of a pitchfork bifurcation 24]), and, concurrently, $g_{1}$ turns from circulation to libration.

For s-III, $e_{1}$ initially oscillates, but it momently vanishes at $a_{2} \sim 4800$. Subsequently, $e_{1}$ starts to increase, and $g_{1}$ settles into libration. In Fig. 1A, the evolution of the system s-II is similar to s-I. Therefore, the basic aspects of the transition could be understood by analyzing the simpler system s-I with $e_{2}=0$ (as we limit below). In common with the three systems, the mean values of $e_{1}$ evolve slowly with time.

It has been known that, for $e_{2} \neq 0$, the inner eccentricity $e_{1}$ can experience a resonant-like excitation around the epoch with $T_{\mathrm{KL}} \sim T_{1 \mathrm{PN}}[9,10]$. Recently, Liu, Lai and Yuan 25] reported that this excitation is caused by an apsidal precession resonance $\dot{\varpi}_{1}-\dot{\varpi}_{2} \simeq 0$. Here, $\varpi_{i}\left(\equiv \Omega_{i}+g_{i}\right)$ is the longitude of the pericenter and $\Omega_{i}$ is the longitude of the ascending node.

Our primary target in this paper is the inner orbital evolution for $e_{2}=0$. But, here, we briefly comment on this resonance. For the run s-II mentioned above, we have $\dot{\varpi}_{1} \gg \dot{\varpi}_{2}$ due to the hierarchies of the masses and orbits. Therefore, the resonant state $\dot{\varpi}_{1}-\dot{\varpi}_{2} \simeq 0$ was not realized in the run.

In the next section, we study a more realistic system including a stellar cluster potential with $e_{2}=0$. It would be interesting to examine the effects of the apsidal precession resonance for $e_{2} \neq 0$. But, such an extension is far beyond the scope of this paper, since we need additional inputs, e.g. a detailed modeling of the outer precession rate $\dot{\varpi}_{2}$. We remain this issue as a future work. 


\section{B. Averaged Hamiltonian}

To geometrically explain the interesting behaviors in Fig. 1, we briefly describe the employed Hamiltonian. For studying the long-term evolution of the triples, we take the double averages of the original three-body Hamiltonian with the inner and outer mean anomalies [9, 26]. After appropriate scaling, we obtain the relevant Hamiltonian [10, 18]

$$
\mathcal{H}_{\mathrm{T}, 1 \mathrm{PN}}=\mathcal{H}_{\mathrm{qp}}+\mathcal{H}_{1 \mathrm{PN}}
$$

with

$$
\mathcal{H}_{\mathrm{qp}} \equiv-3 G_{1}^{2}-15 \frac{J_{1}^{2}}{G_{1}^{2}}-15\left(1-G_{1}^{2}\right)\left(1-\frac{J_{1}^{2}}{G_{1}^{2}}\right) \cos 2 g_{1},
$$$$
\mathcal{H}_{1 \mathrm{PN}} \equiv-\frac{3 \gamma}{G_{1}}
$$

Here, $\mathcal{H}_{\mathrm{qp}}$ is the Newtonian quadrupole term due to the outer tertiary, and is obtained by perturbatively evaluating the tidal field of the tertiary $m_{2}$ with the expansion parameter $\left(a_{1} / a_{2}\right) \ll 1$. The subsequent-order (octupole) term vanishes for $e_{2}=0$. The $1 \mathrm{PN}$ term $\mathcal{H}_{1 \mathrm{PN}}$ leads to an apsidal precession of the inner orbit [16].

The dynamical degree of freedom of this Hamiltonian is only the canonical variables $\left(g_{1}, G_{1}\right)$. Below, as supported by the numerical experiments, we put $a_{1}=$ const and $J_{1}=$ const (ignoring the inner radiation reaction) 2 . The decreasing parameter $\gamma$ is defined as

$$
\gamma \equiv \frac{16\left(m_{0}+m_{1}\right)^{2} a_{2}^{3}}{\left(m_{2} a_{1}^{4}\right)} \propto \frac{a_{2}^{3}}{a_{1}^{4}}\left(\propto \frac{T_{\mathrm{KL}}}{T_{1 \mathrm{PN}}}\right),
$$

and contains the information of the outer orbits in $\mathcal{H}_{\mathrm{T}, 1 \mathrm{PN}}$. The $1 \mathrm{PN}$ effect gradually becomes weaker, relative to the tidal field of the infalling tertiary.

In the $\left(g_{1}, G_{1}\right)$ coordinate, the angle variable $g_{1}$ is singular at $G_{1}=J_{1}$ and 1 , since $g_{1}$ loses its geometrical meanings there (either $I=0$ or $e_{1}=0$ ). These coordinate singularities should be regarded as two degenerate points, and can be appropriately handled with the canonical transformations [4];

$$
\begin{array}{r}
\left(x^{\prime}, y^{\prime}\right)=\sqrt{2\left(G_{1}-J_{1}\right)}\left(\cos g_{1}, \sin g_{1}\right) \\
(x, y)=\sqrt{2\left(1-G_{1}\right)}\left(\cos g_{1}, \sin g_{1}\right)
\end{array}
$$

As expected from geometric symmetry, these two points are fixed points in the phase space, irrespective of the parameter $\gamma$.

\footnotetext{
${ }^{2}$ Also, our Hamiltonian $\mathcal{H}_{\mathrm{T}, 1 \mathrm{PN}}$ is independent of their conjugate variables.
}

\section{Geometrical approach}

For our geometrical approach, we need to understand how the phase-space structure changes along with the slowly decreasing parameter $\gamma$. From general arguments on Hamiltonian systems, a fixed point must be either a center (stable fixed point) or saddle (unstable fixed point) [24].

In Fig. 2, we show the phase-space structure of the Hamiltonian $\mathcal{H}_{\mathrm{T}, 1 \mathrm{PN}}\left(g_{1}, G_{1} ; \gamma\right)$ for $\gamma=14.89,10.03$ and 7.37. First, we focus on the solid black curves (contour samples of $\mathcal{H}_{\mathrm{T}, 1 \mathrm{PN}}$ ), the black points (unstable fixed points), and the red broken curves (separatrixes). These elements evolve in the following order.

(P1) For $\gamma>12-20 J_{1}^{2} \equiv \gamma_{\mathrm{c}}$ (Fig. 2A), we only have circulating trajectories. Accordingly, the fixed points at $G_{1}=1$ and $J_{1}$ are both stable. The latter remains stable during whole.

(P2) At the incident $\gamma=\gamma_{\mathrm{c}}$ (between Figs. 2A and $2 \mathrm{~B}$ ), the fixed point $G_{1}=1$ transits from a stable point to an unstable point (pitchfork bifurcation [24]). For $J_{1}=0.150$, this bifurcation is at $\gamma_{\mathrm{c}}=11.55$ (nicely corresponding to $a_{2} \simeq 5238$ in Fig. $1 \mathrm{~A}$ ).

(P3) For $\gamma<\gamma_{\mathrm{c}}$ (Figs. 2B and 2C), we simultaneously have circulating and librating trajectories, segregated by the separatrixes. As $\gamma$ decreases, the stable points at $g_{1}=$ $\pm \pi / 2$ take smaller $G_{1}$. The area inside the separatrixes increases monotonically.

Then, how can we trace the evolution of individual systems in the sequence of Figs.2A-2C? Here, we explain a powerful tool. Let us consider a periodic motion described by a Hamiltonian $\mathcal{H}(q, p ; \lambda)$, including a parameter $\lambda$. If the parameter $\lambda$ changes more slowly than the period of the motion, the area surrounded by the periodic trajectory $S \equiv \oint p d q$ is conserved and called an adiabatic invariant 26,27 .

Now, let us trace the evolution of the system s-III in the phase-space, based on the adiabatic invariant. As in Fig. $2 \mathrm{~A}$, this system initially circulates in the $\left(g_{1}, G_{1}\right)$ plane, but it is periodic in the $(x, y)$ plane with the calculated adiabatic invariant $S=0.129 \equiv S_{\mathrm{III}}$. Here the area $S_{\mathrm{III}}$ corresponds to the blue region in Fig. 2A. Because of the area conservation, at the stage of Fig. 2B, s-III is expected to stay circulating on the thick blue curve.

But, as $\gamma$ decreases, the total area inside the two separatrixes increases, and it eventually becomes identical to $S_{\text {III }}$ between Fig. 2B and 2C (around $\gamma \sim 8.89$ ). At the separatrix crossing, $e_{1}$ momently vanishes, and the system s-III starts to librate either around $g_{1}=\pi / 2$ or $-\pi / 2$ (equal probability from the symmetry), due to the continuity of the trajectory. Associated with this disjunction, the adiabatic invariant $S$ falls to one-half $S_{\mathrm{III}} / 2$. But this gap is not contracting with the adiabatic invariance, since the period becomes infinite for the trajectory just on the separatrix. After crossing the separatrix, we can again follow the system, using the area conservation around one of the two stable points. For example, the averaged value of $e_{1}$ increases with time. In this manner, we can 


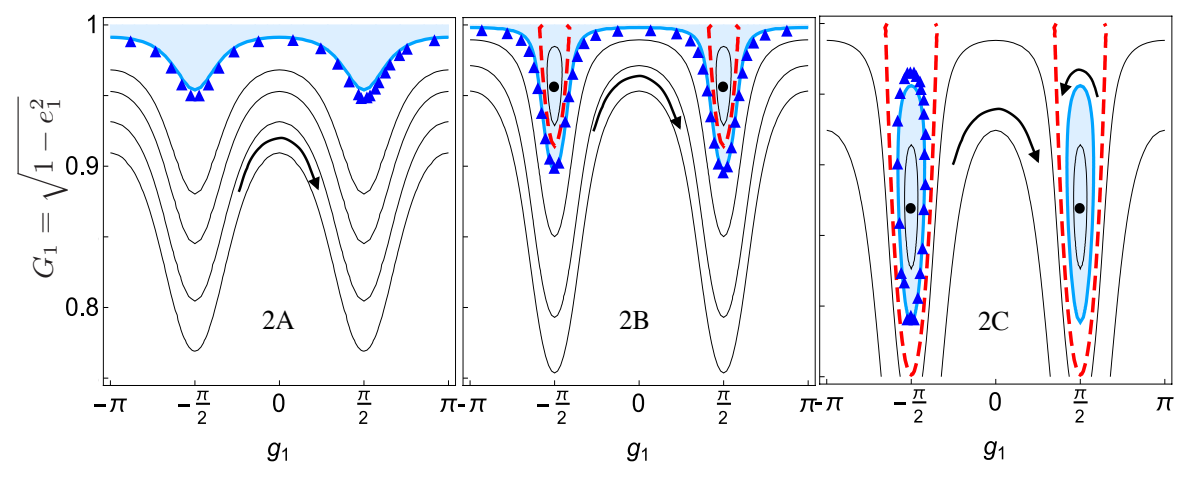

FIG. 2. PN evolution of the phase-space of the inner binary with $J_{1}=0.150$ (plotted in the limited range $0.75 \leq G_{1} \leq 1$ ). Figs. 2A, 2B and 2C correspond to $\gamma=14.88,10.03$ and 7.37 (decreased by the tertiary). The following two types of information are presented simultaneously in each panel. [1. the overall phase-space structure of the Hamiltonian $\mathcal{H}_{\mathrm{T}, 1 \mathrm{PN}}$ ] solid black curves: contour samples of $\mathcal{H}_{\mathrm{T}, 1 \mathrm{PN}}$, black points: stable fixed points, red dashed curves: separatrixes, and arrows: directions of flows. [2. evolution of the specific system s-III] The blue regions have equal area $S_{\mathrm{III}}=0.129$ (corresponding to the adiabatic invariant). The triangles represent the results from the direct three-body calculation for s-III.

geometrically understand the evolution of s-III.

Until now, we have discussed the phase-space mapping of the specific system s-III. Generally, in the mapping from Fig.2A to $2 \mathrm{C}$, a system initially closer to $G_{1}=1$ is transported to a librating trajectory closer to one of the two stable fixed points (crossing the separatrix earlier). As the present mapping is thus simple and symmetric, we can justify the area conservation also by applying Liouville's theorem to certain group of systems (e.g. these composing the blue region in Fig. 2A). Indeed, in Fig. 2, the total area of the blue region is the same, before and after the separatrix crossing.

Next, we examine the validity of our approach, using the direct three-body calculations. In Fig. 2, the triangles represent the numerical results for s-III. The associated area is conserved at high precision. Assuming the area conservation, we can inversely predict the evolution of $e_{1}$ easily. In Fig. 1B, with the blue points, we represent the maximum and minimum values of $e_{1}$ at each $a_{2}$. They well reproduce the direct calculations 3 .

In the case of s-I (with much smaller area $S_{\mathrm{I}} \ll S_{\text {III }}$ ), the trajectory turns into libration soon after (P2) and it stays close to the stable fixed point throughout Fig. $2 \mathrm{~B}$ and $2 \mathrm{C}$. This is why the distinctive features of the pitchfork bifurcation (originally associated with the fixed points) appear in Fig. 1A.

\section{NEWTONIAN POTENTIAL OF STELLAR CLUSTERS}

We next apply our approach to the evolution of individual stars in a stellar cluster around a central $\mathrm{MBH} m_{0}$

${ }^{3}$ At the very end of the run, there exist mismatches caused by breaks-down of the averaging method for $e_{1} \sim 1$ [5, 15, 28]. with an outer infalling tertiary $\mathrm{MBH} m_{2}$. We deal with component stars for which (i) the dominant force is the Newtonian attraction by the central $\mathrm{MBH} m_{0}$, but (ii) its 1PN correction would be smaller than the Newtonian potential of the cluster itself. Here, we ignore the $1 \mathrm{PN}$ effect for simplicity, but it could play important roles for stars with small pericenter distances (see e.g. [3]). In this section, we consider outer $\mathrm{MBH}$ that have larger separation (still on circular orbit) than the previous PN systems (see §III.B for parameters in the physical units). We do not specify the physical processes for the outer orbital decay, but the infall rate is assumed to be sufficiently small (e.g. after the stall of dynamical friction [3]). We also disregard dissipative effects for the inner orbit (i.e. setting $a_{1}=$ const and $J_{1}=$ const). The relaxation processes would be discussed in §III.B (see e.g. $[6,29,[30])$.

\section{A. Geometrical approach}

We first present the averaged Hamiltonian. The cluster is initially assumed to be spherical with the local density profile $\rho(r)=\rho_{1}\left(r / a_{1}\right)^{-\beta}(\beta<3)$ around the distance $r=a_{1}$ in interest $\left(\rho_{1}\right.$ : the mean density there). Ignoring the back-reaction (time variation) to the stellar potential, the scaled Hamiltonian is written as

$$
\mathcal{H}_{\mathrm{T}, \mathrm{SP}}=\mathcal{H}_{\mathrm{qP}}+\mathcal{H}_{\mathrm{SP}} .
$$

Here, $\mathcal{H}_{\mathrm{SP}}$ is the term due to the stellar potential

$$
\mathcal{H}_{\mathrm{SP}}=\eta e_{1}^{2}\left[1+\beta(-1+\beta)\left(\frac{e_{1}^{2}}{16}+\mathcal{O}\left(e_{1}^{4}\right)\right)\right],
$$

which is obtained by perturbatively expanding a hypergeometric function with $e_{1}=\sqrt{1-G_{1}^{2}}[3]$. In our geometric approach, we neglect the small higher-order correction $o\left(e_{1}^{2}\right)$ that vanishes identically at $\beta=0$ and 1 (though 
exactly handled later with $\beta=3 / 2$ ). Namely, we put $\mathcal{H}_{\mathrm{SP}}=\eta\left(1-G_{1}^{2}\right)$ in terms of the canonical variable $G_{1}$. Then, all the information of the cluster is included in the single decreasing parameter

$$
\eta \equiv \frac{16 \pi \rho_{1} a_{2}^{3}}{m_{2}}
$$

with $\dot{a}_{2} \equiv \partial_{t} a_{2}<0$.

The precession timescale due to the stellar potential $T_{\mathrm{SP}}[3]$ is

$$
T_{\mathrm{SP}}=\frac{(3-\beta) m_{0}}{4 \pi \rho_{1} a_{1}^{3}} P_{1} .
$$

Then, we can represent $\eta$ by using $T_{\mathrm{KL}}$ and $T_{\mathrm{SP}}$ as

$$
\eta=\frac{12 \pi}{3-\beta} \frac{T_{\mathrm{KL}}}{T_{\mathrm{SP}}} .
$$

Next, we explain the evolution of the phase-space structure, following Fig. 3 (see also [4]). The most remarkable point below is the bifurcation (P4') at $\eta_{\mathrm{c} 2} \equiv 12$ (independent of $J_{1}$ !).

(P1') For $\eta>-18+30 / J_{1}^{2} \equiv \eta_{\mathrm{c} 1}$ (Fig. 3A), we only have circulating trajectories in the retrograde direction.

(P2') At $\eta=\eta_{\mathrm{c} 1}$ (between Figs. 3A and 3B), we have a pitchfork bifurcation similar to (P2). The fixed point $G_{1}=J_{1}$ turns from a stable point to an unstable point ( in contrast to $G_{1}=1$ for (P2), see also Appendix A). The new stable point (black point in Fig. 3B) is generated at $g_{1}=\pi / 2$.

(P3') For $\eta_{\mathrm{c} 2} \equiv 12<\eta<\eta_{\mathrm{c} 1}$ (Fig. 3B), we have librating trajectories inside the separatrix (red dashed curve). The inner area increases with time.

(P4') At $\eta=\eta_{\mathrm{c} 2}$ (Fig. 3C), due to a cancellation between $\mathcal{H}_{\mathrm{qp}}$ and $\mathcal{H}_{\mathrm{SP}}$, the Hamiltonian $\mathcal{H}_{\mathrm{T}, \mathrm{SP}}$ becomes independent of $G_{1}$ on the two vertical lines $g_{1}=0$ and $\pi$. This is a quite peculiar state in which fixed points spread fully on the two lines. At this moment, the stability of the two points $G_{1}=J_{1}$ and 1 interchange, and only librating trajectories exist.

(P5') For $\eta_{\mathrm{c} 3} \equiv-18+30 J_{1}^{2}<\eta<\eta_{\mathrm{c} 2}$ (Fig. 3D), we again have both librating and circulating trajectories. The latter now have prograde motion. The area inside the separatrix decreases.

For $J_{1}>\sqrt{3 / 5}$, we have $\eta_{\mathrm{c} 3}>0$. Thus, if $\eta$ decreases down to $\eta_{\mathrm{c} 3}$, the stable point at $g_{1}=\pi / 2$ disappears, and the fixed point $G_{1}=1$ turns to be stable (inverse of the bifurcation at (P2)). We then have only circulating trajectories.

Now, using the area conservation, we follow the evolution of two representative stars s-IV and $s-\mathrm{V}$ in the time sequence of Fig. 3. Their initial conditions $\left(g_{1}\right.$, $\left.G_{1}\right)$ at $\eta=200$ are $(0,0.95)$ for s-IV, and $(0,0.6)$ for s$\mathrm{V}$ both with $J_{1}=0.5$. In Fig. $3 \mathrm{~A}$, we can effectively regard the areas of the colored regions (blue: s-IV and green: $\mathrm{s}-\mathrm{V}$ ) as conserved quantities. Conserving these areas, the two systems would evolve as shown with the thick blue/green curves in Figs. 3B-3D. Note that, at the first separatrix crossing during (P3'), the maximum eccentricity (i.e. $G_{1}=J_{1}$ ) is surely realized (in contrast to $G_{1}=1$ for the $1 \mathrm{PN}$ case).

Interestingly, the magnitude of their eccentricities is inverted between Fig. 3A and 3D. This is because of the passage of the peculiar state (P4'). Consequently, nearly circular orbits $\left(G_{1} \sim 1\right)$ would be quickly converted to eccentric ones $\left(G_{1} \sim J_{1}\right)$ around $\eta=12$. This sharp transition is quite different from the more gradual evolution in the PN case, and would be less likely to be prevented by other processes.

If the cluster initially has an isotropic velocity distribution, the stars accordingly have a homogeneous density distribution in the $\left(g_{1}, G_{1}\right)$ plane [31]. Then, because of Liouville's theorem, the homogeneity is unchanged in the subsequent stages in Fig. 3, and our assumption about the stationarity of the cluster potential would be a good approximation. Meanwhile, for an initial distribution biased to small eccentricities, the secure realization of the maximum eccentricity during (P3') could help to refill the loss cone, and might be very important astrophysically.

In Fig. 3, we additionally present the results obtained by numerically solving the Hamilton equations;

$$
\dot{G}_{1}=-\frac{\partial \mathcal{H}_{\mathrm{T}, \mathrm{SP}}}{\partial g_{1}}, \quad \dot{g}_{1}=\frac{\partial \mathcal{H}_{\mathrm{T}, \mathrm{SP}}}{\partial G_{1}}
$$

with $\beta=3 / 2$ and $\dot{\eta}=-10^{-3}$ (including $o\left(e_{1}^{2}\right)$-term for $\left.\mathcal{H}_{\mathrm{SP}}\right)$. Except for some fluctuations around $\eta=12$, our simple geometrical predictions fit the numerical results well.

\section{B. Effects of Relaxation}

Until now, we have neglected the diffusion of the energy and angular momentum associated with the discreteness of the stellar distribution. In this subsection, we estimate the characteristic timescales of the relevant relaxation processes.

\section{Model parameters}

To begin with, we set the fiducial model for the overall cluster density profile as

$$
\rho(r)=\frac{3 m_{0}}{4 \pi r_{\mathrm{h}}^{3}}\left(\frac{r}{r_{\mathrm{h}}}\right)^{-3 / 2},
$$

with the slope $\beta=3 / 2$ [32]. Here $r_{\mathrm{h}}$ is the influenced radius $r_{\mathrm{h}}=G m_{0} / \sigma^{2}$ with the one-dimensional velocity dispersion $\sigma$ that is assumed to satisfy the $M_{\bullet}-\sigma$ relation 33];

$$
M_{\bullet}\left(=m_{0}\right)=2.09 \times 10^{8}\left(\frac{\sigma}{200 \mathrm{~km} / \mathrm{s}}\right)^{5.64} M_{\odot} .
$$




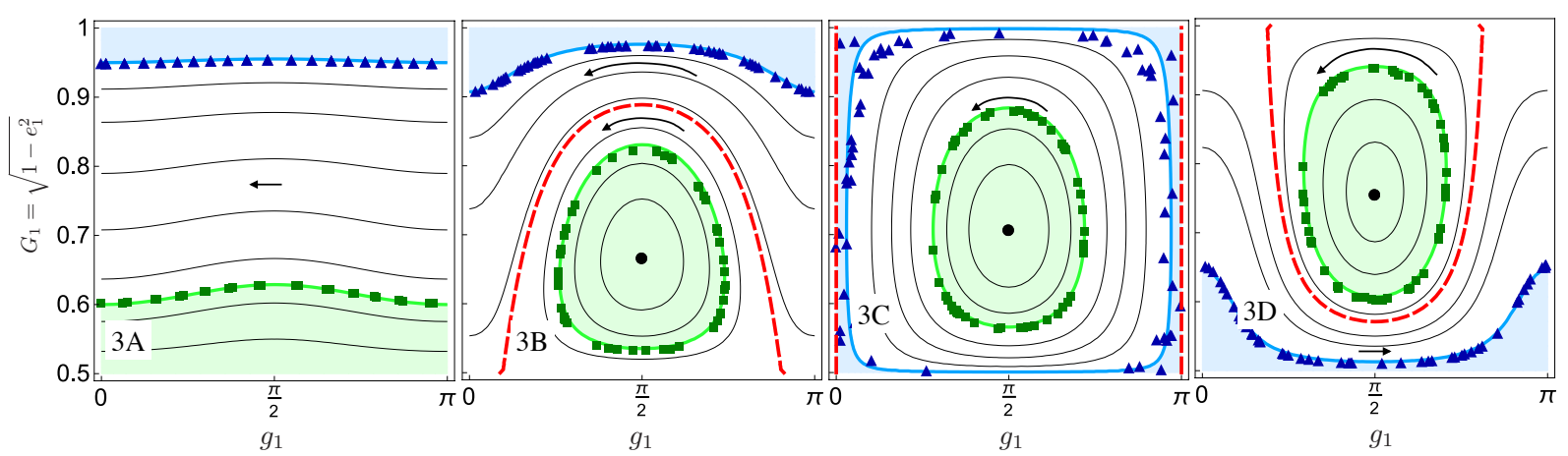

FIG. 3. Evolution of the phase-space for stars in a stellar cluster around a MBH (fixed at $J_{1}=0.50$ ). Figures $3 \mathrm{~A}, 3 \mathrm{~B}, 3 \mathrm{C}$ and $3 \mathrm{D}$ are for $\eta=200,20,12$ and 5 decreased by an infalling tertiary MBH. Because of the symmetry, we only plot the range $0 \leq g_{1} \leq \pi$. As in Fig. 2, the following two types of information are simultaneously plotted. [1. the phase-space structure of $\left.\mathcal{H}_{\mathrm{T}, \mathrm{SP}}\right]$ The symbols (solid black curves, black dots, red dashed curves and arrows) are defined in the same manner as Fig. 2. [2. evolution of the two representative stars s-IV (blue) and s-V (green)]. The areas of the colored regions are respectively the same. The triangles and squares are obtained by numerically integrating the Hamilton equations with $\beta=3 / 2$ and $\dot{\eta}=-10^{-3}$.

From Eqs. (15) and (18), the timescale $T_{\mathrm{SP}}$ of the mass precession is given by

$$
T_{\mathrm{SP}}=2.58 \times 10^{5}\left(\frac{m_{0}}{10^{8} M_{\odot}}\right)^{0.47} \mathrm{yr}
$$

without depending on $a_{1}$ (for $\beta=3 / 2$ ).

In the standard picture of MBH binary evolution [3], the orbital decay timescale $T_{\mathrm{dec}} \equiv a_{2} / \dot{a}_{2}$ is considered to increase sharply around the hard-binary separation

$$
\begin{aligned}
a_{\mathrm{h}} & \equiv \frac{m_{2}}{m_{0}+m_{2}} \frac{r_{\mathrm{h}}}{4} \\
& =3.50 \frac{q}{q+1}\left(\frac{m_{0}}{10^{8} M_{\odot}}\right)^{0.645} \mathrm{pc}
\end{aligned}
$$

with $q \equiv m_{2} / m_{0}<1$. The subsequent orbital decay would strongly depend on the exterior of the MBH binary (e.g. triaxial potential [34, 35]), and the actual timescale $T_{\mathrm{dec}}$ is still under active discussion. We are specifically interested in the orbits of stars around the peculiar bifurcation at $\eta=12$. For applying the adiabatic invariance, the decay timescale $T_{\mathrm{dec}}$ must be smaller than the relaxation timescales, but larger than $T_{\mathrm{KL}}$ that is explicitly given as

$$
\begin{aligned}
T_{\mathrm{KL}}= & \frac{5.45 \times 10^{5}}{q}\left(\frac{m_{0}}{10^{8} M_{\odot}}\right)^{-1 / 2} \\
& \times\left(\frac{a_{1}}{0.3 \mathrm{pc}}\right)^{-3 / 2}\left(\frac{a_{2}}{3.5 \mathrm{pc}}\right)^{3} \mathrm{yr} .
\end{aligned}
$$

\section{Resonant Relaxations}

Now, we discuss the timescales for the relaxation processes. As is well-known for nuclear star clusters, the timescale for the two-body relaxation is generally much larger than that for the resonant relaxation. Therefore, in the following, we concentrate on the resonant relaxation.

We firstly summarize the basic aspects of the resonant relaxation for a conventional system composed only by a central MBH and the associated stellar clusters [3]. Then, we discuss how the resonant relaxation would be modified by the outer $\mathrm{MBH} m_{2}$.

In a spherical stellar cluster around a central $\mathrm{MBH}$ $m_{0}$, the individual stars maintain their Keplerian orbits for the precession timescale $T_{\mathrm{SP}}$. The scalar resonant relaxation is the long-term accumulation of the gravitational interaction between these Keplerian orbits of the short-coherence time $T_{\mathrm{SP}}$. Both the magnitude and orientation of the angular momenta of the stars change with the characteristic timescale [3]

$$
\begin{aligned}
T_{\mathrm{rr}, \mathrm{s}} & =\frac{m_{0}}{m_{1}} P_{1} \\
& =1.6 \times 10^{9}\left(\frac{1 M_{\odot}}{m_{1}}\right)\left(\frac{m_{0}}{10^{8} M_{\odot}}\right)^{1 / 2}\left(\frac{a_{1}}{0.3 \mathrm{pc}}\right)^{3 / 2} \mathrm{yr} .
\end{aligned}
$$

Meanwhile, the vector resonant relaxation should be regarded as interaction between the mass annulus obtained after the orbital averaging over the precession timescale $T_{\mathrm{SP}}$. It changes only the direction of the angular momentum (due to the symmetry) with the characteristic time [3]

$$
T_{\mathrm{rr}, \mathrm{v}} \sim \frac{T_{\mathrm{rr}, \mathrm{s}}}{\sqrt{N}}
$$

where $N$ is the number of stars around the semimajor axis in interest.

Next, we discuss the impacts of the additional outer $\mathrm{MBH} m_{2}$ on the two-types of resonant relaxations. Now, the individual Keplerian orbits maintain their shapes for $\min \left[T_{\mathrm{KL}}, T_{\mathrm{SP}}\right]$. Therefore, for $T_{\mathrm{KL}} \lesssim T_{\mathrm{SP}}$, the timescale 
of the scalar resonant relaxation would be a factor of $T_{\mathrm{SP}} / T_{\mathrm{KL}}=(\eta / 12)^{-1} \pi /(3-\beta)$ times larger than $T_{\mathrm{rr}, \mathrm{s}}$ in Eq.(23). This factor is of order unity for $\eta \sim 12$,

Here, it is important to note that the outer $\mathrm{MBH}$ also generates precession of the longitude of the ascending node $\Omega_{1}$ of the stars with the characteristic timescale $T_{\mathrm{KL}}$ (because $\left.\left|\dot{\Omega}_{1}\right|=\left|\partial \mathcal{H}_{\mathrm{T}, \mathrm{SP}} / \partial J_{1}\right| \sim\left|\partial \mathcal{H}_{\mathrm{T}, \mathrm{SP}} / \partial G_{1}\right|=\left|\dot{g}_{1}\right|\right)$. Therefore, around $\eta \sim 12$, the vector resonant relaxation should be regarded as gravitational interaction between axisymmetric (torus-like) mass distributions with the common symmetric axis (rather than the inclined annulus for the conventional case). But there is no transfer of torque between them (due to the symmetry), and, consequently, the vector resonant relaxation becomes ineffective around the regime $\eta \sim 12$.

We conclude that, even though the decay timescale $T_{\mathrm{dec}}=a_{2} / \dot{a}_{2}$ is uncertain at $a_{2}<a_{\mathrm{h}}$, it should be smaller than $T_{\mathrm{rr}, \mathrm{s}}$ in Eq.(23) for applying the adiabatic invariance to the bifurcation around $\eta=12$.

\section{DISCUSSIONS}

We have developed a geometrical approach for the adiabatic evolution of hierarchical triple systems including MBH binaries on circular orbits. We analyzed the basic profile (e.g. fixed points and separatrixes ) of the phase- space structure of the corresponding Hamiltonian. In addition, we apply the arguments of the adiabatic invariance to follow the time evolution of individual systems.

At first, we demonstrated the validity of our approach using a simple relativistic systems that can be also examined by direct numerical calculations. Then, we analyzed more realistic systems, star clusters associated with inspiraling $\mathrm{MBH}$ binaries. We found that the eccentricities of the stars could evolve quite interestingly. This is caused by the peculiar bifurcation patterns induced by the Newtonian potential of stellar clusters, in sharp contrast to the precedent relativistic systems. Our simple geometrical approach enables us to clearly understand how the inner orbits are deformed, in response to the time variation of the related phase-space structure. It would be applicable to the KL mechanism in various astrophysical contexts, possibly including viscous effects to the inner orbit at least for a short time.

\section{ACKNOWLEDGMENTS}

We would like to thank M. Iwasawa, H. Nakano and A. Tanikawa for helpful conversations. This work was supported by JSPS $(24540269,15 \mathrm{~K} 05075)$ and MEXT (24103006).
[1] P. Amaro-Seoane et al., GW Notes 6, 4 (2013).

[2] X. Chen, A. Sesana, P. Madau and F. K. Liu, Astrophys. J.729, 13 (2011).

[3] D. Merritt, Dynamics and Evolution of Galactic Nuclei (Princeton University Press, 2013).

[4] P. B. Ivanov, A. G. Polnarev and P. Saha, Mon. Not. Roy. Astron. Soc. 358, 1361 (2005).

[5] J. N. Bode and C. Wegg, Mon. Not. Roy. Astron. Soc. 438, 573 (2014).

[6] G. Li et al, Mon. Not. Roy. Astron. Soc. 451, 1341 (2015).

[7] Y. Kozai, Astron. J. 67, 591 (1962); M. L. Lidov, Planet.Space Sci. 9,719 (1962).

[8] M. Holman, J. Touma and S. Tremaine, Nature (London)386, 254 (1997).

[9] E. B. Ford, B. Kozinsky and F. A. Rasio, Astrophys. J.535, 385 (2000).

[10] S. Naoz, B. Kocsis, A. Loeb and N. Yunes, Astrophys. J.773, 187 (2013).

[11] B. J. Shappee and T. A. Thompson, Astrophys. J.766, 64 (2013).

[12] E. Michaely and H. B. Perets, Astrophys. J.794, 122 (2014).

[13] A. T. Sinclair, Mon. Not. Roy. Astron. Soc. 160, 169 (1972); J. Henrard, 1982, CeMec 27, 3 (1982); N. Borderies and P. Goldreich, 1984, CeMec 32, 127 (1984); C. D. Murray and S. F. Dermott, Solar System Dynamics (Cambridge University Press, 1999).

[14] L. Wen, Astrophys. J.598, 419 (2003).

[15] B. Katz and S. Dong, arXiv:1211.4584.

[16] D. Fabrycky and S. Tremaine, Astrophys. J.669, 1298
(2007).

[17] S. Naoz and D. C. Fabrycky, Astrophys. J.793, 137 (2014).

[18] O. Blaes, M. H. Lee and A. Socrates, Astrophys. J.578, 775 (2002).

[19] T. A. Thompson, Astrophys. J.741, 82 (2011).

[20] D. J. Muñoz and D. Lai, arXiv:1505.05514.

[21] P. Jaranowski and G. Schäfer, Phys. Rev. D55, 4712 (1997).

[22] R. A. Mardling and S. J. Aarseth, Mon. Not. Roy. Astron. Soc. 321, 398 (2001).

[23] P. C. Peters, Phys. Rev. 136, B1224 (1964).

[24] S. H. Strogatz, Nonlinear Dynamics and Chaos (Addison-Wesley, 1994).

[25] B. Liu, D. Lai, and Y.-F. Yuan, Phys. Rev. D. 92, 124048 (2015)

[26] H. Goldstein, Classical Mechanics, second edition (Addison-Wesley, 1980).

[27] L. D. Landau and E. M. Lifshitz, Mechanics (Pergamon Press, Oxford, 1969).

[28] F. Antonini, N. Murray and S. Mikkola, Astrophys. J.781, 45 (2014). J. M. Antognini, B. J. Shappee, T. A. Thompson and P. Amaro-Seoane, Mon. Not. Roy. Astron. Soc. 439, 1079 (2014); N. Seto, Phys. Rev. Lett. 111, 061106 (2013).

[29] T. Alexander, Phys. Rept. 419, 65 (2005).

[30] K. P. Rauch, and S. Tremaine, New Astron. 1, 149 (1996).

[31] J. Binney and S. Tremaine, Galactic Dynamics (Princeton University Press, 2008). 
[32] R. M. O'Leary, and A. Loeb, Mon. Not. Roy. Astron. Soc. 395, 781 (2009).

[33] N. J. McConnell, and C.-P. Ma, Astrophys. J., 764, 184 (2013).

[34] F. M. Khan, A. Just, and D. Merritt, Astrophys. J., 732, 89 (2011)

[35] E. Vasiliev, F. Antonini, and D. Merritt, Astrophys. J., 785, 163 (2014)

\section{Appendix A: Generation of a New Fixed Point}

As shown in Figs. 2 and 3, the phase-space evolution is largely different between the two Hamiltonians $\mathcal{H}_{\mathrm{T}, 1 \mathrm{PN}}$ and $\mathcal{H}_{\mathrm{T}, \mathrm{SP}}$ given in Eqs. (6) and (12). When relatively increasing the quadrupole term (i.e. $\gamma, \eta=+\infty \rightarrow 0$ ), one of the remarkable contrasts is the emergence of the new fixed point either at $G_{1}=1$ (the epoch (P2) for $1 \mathrm{PN}$ ) or at $G_{1}=J_{1}$ (the epoch $\left(\mathrm{P} 2^{\prime}\right)$ for $\mathrm{SP}$ ) both with $g_{1}= \pm \pi / 2$. In this appendix, we discuss how this contrast is related to the sign of the apsidal precession $(1 \mathrm{PN}$ : prograde and SP: retrograde).

In the present case, the condition for the fixed point is formally expressed as

$$
\left.\frac{\partial \mathcal{H}_{\mathrm{T}}}{\partial G_{1}}\right|_{g_{1}=\pi / 2}=0
$$

for $\mathcal{H}_{\mathrm{T}}=\mathcal{H}_{\mathrm{T}, 1 \mathrm{PN}}$ and $\mathcal{H}_{\mathrm{T}, \mathrm{SP}}$. This equation is physically equivalent to $\dot{g}_{1}=0$ for the apsidal precession rate, representing the balance between the quadrupole effect and the competing $1 \mathrm{PN}$ or SP effect. In concrete terms, we collectively have

$$
Q\left(G_{1}\right) \equiv-36 G_{1}+60 \frac{J_{1^{2}}}{G_{1}^{3}}=-k G_{1}^{-\alpha}
$$

Here, the function $Q\left(G_{1}\right)$ originates from the quadrupole term and monotonically decreases in the relevant range $J_{1} \leq G_{1} \leq 1$. The right hand side shows the apsidal precession rates induced by the $1 \mathrm{PN}(\alpha=2$ and $k(=$ $3 \gamma)>0)$ and the SP $(\alpha=-1$ and $k(=-2 \eta)<0)$.

Now, for simplicity, we assume $J_{1}<\sqrt{3 / 5}$ (identical to the condition for the standard KL-libration). Then, we have $Q\left(G_{1}=J_{1}\right)>0$ and $Q\left(G_{1}=1\right)<0$. Furthermore, with respect to the two types of variations $k=+\infty \rightarrow 0$ or $k=-\infty \rightarrow 0$, either of two solutions; $G_{1}=J_{1}$ or $G_{1}=1$ initially appears in Eq. A2 , irrespective of the index $\alpha \in[-1,2]$. Indeed, depending only on the sign of $k$, we have $G_{1}=1$ (for $k=+\infty \rightarrow 0$ ) and $G_{1}=J_{1}$ (for $k=-\infty \rightarrow 0)$.

This result explains the sharp contrast between (P2) and (P2'). Here, the sign of the apsidal precession is crucially important. Note also that, even if a small correction term is added to the right hand side of Eq.(A2), this robust result is unchanged. 